\section{PSQ-057 ANALYSIS OF THE INCIDENCE OF HEPATOTOXICITY ASSOCIATED WITH THE USE OF TOCILIZUMAB}

L Cantarelli*, E Ramos Santana, F Gutierrez Nicolas, B Del Rosario Garcia, J Ramos Rodriguez, J Garcia Cairos, J Gonzalez Garcia, GA Gonzalez De La Fuente, JA Morales Barrios, S Garcia Gil, GJ Nazco Casariego. Complejo Hospitalario Universitario De Canarias, Pharmacy, Santa Cruz De Tenerife, Spain

\subsection{6/ejhpharm-2020-eahpconf.374}

Background and importance Prolonged treatment with tocilizumab has been associated with cases of severe hepatotoxicity with liver failure and hepatitis, characterised by elevated hepatic transaminases (GOT/AST and GPT/ALT).

Aim and objectives To analyse the incidence of elevation of liver enzymes and the presence of severe liver damage in patients treated with tocilizumab, in a third level hospital.

Material and methods A descriptive, observational, 10 year study that included all patients treated with tocilizumab for more than 6 months, from January 2009 to August 2019, was carried out. Patients in whom the drug was used under special conditions of use and those with abnormal transaminase values prior to the start of treatment were excluded. The variables recorded were age, sex and duration of treatment. Liver function values (GOT/AST and GPT/ALT) were analysed every 4 weeks in the first 6 months of treatment and every 12 weeks after 6 months of treatment. Alterations in these values were classified as mild $(1-3 \times$ normal upper limit $(\mathrm{NAL}))$, moderate $(3-5 \times \mathrm{NAL})$ and severe $(>5 \times \mathrm{NAL})$. Data were collected from a database in Excel format.

Results During the study, a total of 135 patients were treated, 84 intravenously and 51 subcutaneously. Fifty-six patients were excluded from the study: 28 for receiving treatment for $<6$ months, 19 for off-indication regimens and 9 for elevation of liver enzymes prior to drug initiation. The study population was 77 patients: $11.7 \% \quad(n=9)$ men and $88.3 \% \quad(n=68)$ women; mean age was 55.13 years (12-83).

Mean duration of treatment was 40.44 months: $48.1 \%$ $(n=37)$ showed alterations in liver parameters during treatment. In the first 6 months of treatment, $22.1 \%$ of patients $(n=17)$ showed an increase in levels $(82.4 \%$ mild $(n=14)$, $11.8 \%$ moderate $(n=2)$ and $5.9 \%$ severe $(n=1))$. After 6 months of treatment, in $44.2 \%$ of cases $(n=34)$ levels increased $(88.2 \%$ mild $(n=30), 11.8 \%$ moderate $(n=4))$.

Conclusion and relevance Our study showed that the rate of liver toxicity in patients treated with tocilizimumab was about $50 \%$. Severe toxicity was identified in only one patient. These results, as indicated by the European Medicines Agency, show the need for liver function monitoring in patients treated with tocilizumab.

\section{REFERENCES AND/OR ACKNOWLEDGEMENTS}

No conflict of interest.

\section{PSQ-058 THE IMPORTANCE OF MONITORING ADVERSE DRUG REACTIONS: DATA FROM THE TREATMENT OF A RARE DISEASE}

1,2 L Cavallo*,${ }^{1,2} \mathrm{C}$ Migliazzi, ${ }^{2} \mathrm{C}$ Borsino, ${ }^{2} \mathrm{M}$ Minischetti, ${ }^{2} \mathrm{C}$ D'angelo. ${ }^{1}$ University of Milan, Department of Pharmacy, Milan, Italy; ${ }^{2}$ Asst Santi Paolo E Carlo, Pharmacy Unit, Milan, Italy

10.1136/ejhpharm-2020-eahpconf.375

Background and importance Idiopathic pulmonary fibrosis (IPF) is a rare disease characterised by scar tissue formation in the lungs. The prevalence is higher in men (20/100 000) than women $(13 / 100000)$. The average age at onset is 66 years. Currently, nintedanib and pirfenidone are used to treat IPF. They have an antifibrotic and anti-inflammatory activity, slowing down the progression of the disease and are subjected to additional monitoring. It is very important to report any suspected adverse drug reaction (ADR) to better understand the efficacy and safety profiles of both of these drugs.

Aim and objectives We analysed the ADRs reported in the year 2018 for pirfenidone and nintedanib in our hospital.

Material and methods Suspected ADRs reported by patients and sent to the national pharmacovigilance network (RNFV) were analysed.

Results Eleven ADRs were recorded for each drug. Considering that the number of patients treated with pirfenidone was 19 and with nintedanib 25, the number of ADRs reported appeared to be quite relevant. Pirfenidone ADR reports were: 5 (45.4\%) skin disorders, such as dermatitis, erythema and photosensitivity; 3 gastrointestinal disorders (27.2\%), such as diarrhoea, nausea, dysgeusia and inappetence; 2 nervous system (18.2\%), with sleepiness and confusion; and in 1 case $(9 \%)$ there was an increase in levels of aspartate aminotransferase, with a probable onset of hepatic alteration.

The reports for nintedanib were $7(63.6 \%)$ for the gastrointestinal system, of which 4 consisted of diarrhoea, and the others asthenia and nausea; and 4 (36.4\%) related to toxic hepatitis, of which 1 was reported as serious.

The pulmonologists, therefore, reduced the daily dose for 12 patients (54.5\%); for 2 patients (9\%) they changed therapy from pirfenidone to nintedanib and for the remaining ones they temporarily suspended treatment (36.4\%).

Conclusion and relevance Initially IPF had been treated with cortisone drugs, azathioprine and cyclophosphamide, while in the past 10 years the development of novel more specific medicines significantly prolonged life expectancy. Nevertheless, it is essential to carry out continuous monitoring of drugs to ensure that patients are treated as effectively and safely as possible. The pharmacist plays a central role in this activity, through direct interaction with patients during dispensing of medicines.

\section{REFERENCES AND/OR ACKNOWLEDGEMENTS}

No conflict of interest.

\section{PSQ-059 PHARMACIST-LED OBSERVATIONAL STUDY ON QUALITY OF LIFE IN RELAPSING-REMITTING MULTIPLE SCLEROSIS: EVIDENCE FROM THE QOSMOS STUDY}

${ }^{1} \mathrm{~V}$ Damuzzo*, ${ }^{2} \mathrm{D}$ Mengato, ${ }^{1} \mathrm{~F}$ Milani, ${ }^{3} \mathrm{~L}$ Agnoletto, ${ }^{4} \mathrm{~N}$ Freddi, ${ }^{5} \mathrm{R}$ Rampazzo. ${ }^{1} \mathrm{School}$ of Hospital Pharmacy, Department of Pharmaceutical and Pharmacological Sciences-University of Padua, Padua, Italy; ${ }^{2}$ Bolzano General Hospital, Hospital Pharmacy, Bolzano, Italy; ${ }^{3}$ Santa Maria Della Misericordia Hospital, Hospital Pharmacy, Rovigo, Italy; ${ }^{4}$ Sant'antonio HospitalAulss 6 Euganea, Department of Neurology, Padua, Italy; ${ }^{5}$ Health Department-Veneto Region, Coordinamento Settore Farmaceutica-Dispositivi E Protesica, Venice, Italy

\subsection{6/ejhpharm-2020-eahpconf.376}

Background and importance Patient reported outcomes (PRO) are increasingly used to evaluate effectiveness of treatments for multiple sclerosis (MS) and they often include an evaluation of health related quality of life (QoL). In 2017, the Italian Society of Clinical Pharmacy and Therapeutics (SIFaCT) and the National Association of Hospital Pharmacy Students 
(ReNaSFO) established a joint action to update existing data on QoL and its correlation with use of disease modifying drugs in Italian patients. The results will be helpful as reference for future studies using PRO.

Aim and objectives The primary endpoint was QoL score in MS patients. Secondary objectives included QoL correlation with pharmacological therapy and clinical characteristics of patients.

Material and methods We designed a multicentre, observational, cross sectional study. Every patient had to complete a questionnaire on QoL (MS-QoL54) and the pharmacist collected the following data: sex, age, MS type, expanded disability status scale (EDSS) and history of pharmacological treatments. The study was approved by an ethic committee in each centre and patients provided signed informed consent. As MS-QoL54 scores were not normally distributed, we used Spearman's correlation test, ANOVA on ranks for multiple comparisons and the Mann-Whitney test for simple comparisons.

Results We enrolled 341 patients from 16 centres (median age 44.1 years; $68.9 \%$ women) with relapsing-remitting MS from May 2018 to June 2019 (median 20 per centre). The composite indexes of physical and mental well being were correlated with each other $(\mathrm{R}=0.826 ; \mathrm{p}<0.001)$ according to a direct proportionality, and both had an inverse correlation with the degree of EDSS disability $(\mathrm{R}=-0.511, \mathrm{p}<0.001$ and $\mathrm{R}=0.344, \mathrm{p}<0.001$, respectively). Although there was no correlation between QoL and route of administration of the drug, we found significantly lower scores for patients treated with teriflunomide compared with other oral drugs (54.24 points vs 67.64 for fingolimod and 78.25 for dimethyl-fumarate; $\mathrm{p}=0.002$ ).

Conclusion and relevance The study achieved primary and secondary endpoints and indicated a relevant decrease in QoL related to physical health associated with teriflunomide, which deserves further investigations. We also demonstrated that joint action by a scientific society and a student association was a valuable method to perform a no profit, multicentre, observational study in real practice.

\section{REFERENCES AND/OR ACKNOWLEDGEMENTS}

1. Horta-Hernadez AM, et al. Health-related quality of life in multiple sclerosis patients treated with disease-modifying therapies. EJHP 2019;26(Suppl 1):A1A311. https://ejhp.bmj.com/content/26/Suppl_1/A131.2

No conflict of interest.

\section{PSQ-060 EFFECTIVENESS AND SAFETY OF USTEKINUMAB IN CLINICAL PRACTICE FOR CROHN'S DISEASE}

${ }^{1}$ M Fages*, ${ }^{2}$ G García Marín, ${ }^{3}$ J Romero Puerto, ${ }^{3}$ A Soria Martín, ${ }^{3}$ MP Quesada Sanz. ${ }^{1}$ Punta De Europa Hospital, Pharmacy, Algeciras, Spain; ${ }^{2}$ Hospital De Europa, Algeciras, Algeciras, Spain; ${ }^{3}$ Hospital Punta De Europa, Farmacia, Algeciras, Spain

10.1136/ejhpharm-2020-eahpconf.377

Background and importance Ustekinumab is a monoclonal antibody that inhibits the bioactivity of IL-12 and IL-23 causing a decrease in inflammatory markers in Crohn's disease (CD), used in patients in whom conventional treatment or anti-TNF is insufficient to control the disease or are contraindicated.

Aim and objectives To evaluate the efficacy and safety of ustekinumab in patients diagnosed with $\mathrm{CD}$ in a real clinical setting.
Material and methods This was a retrospective observational study, in two regional hospitals, in patients with CD who received an induction dose of ustekinumab between January 2018 and September 2019, inclusive. The data were obtained from the PRISMA-APD outpatient care programme, and by reviewing medical records in Diraya. To assess efficacy, the Harvey-Bradshaw index (HBI) was considered, for which the following variables were recorded: general condition of the patient, abdominal pain, number of daily liquid bowel movements, presence or absence of abdominal mass and other associated symptoms. Remission of the disease was considered if HBI was 1-6. Other clinical variables included were: age, sex, previous treatments with anti-TNF, concomitant use with immunomodulators and/or corticosteroids, need for intensification and treatment interruption. To assess safety, adverse effects associated with ustekinumab were considered.

Results Thirty-seven patients were included in the study: 21 women and 16 men. Median age was 45 years. With the exception of three patients, all had received prior therapy with one or more anti-TNF. Twenty of the patients had received concomitant corticosteroid and immunosuppressive medication. In 4 patients ustekinumab was withdrawn due to lack of action but 29 patients presented with an $\mathrm{HBI}<6$, of whom 23 had an intensified pattern (90 mg every 8 weeks). The only adverse reaction recorded was atypical erythema nodosum in a patient.

Conclusion and relevance Ustekinumab seemed to have good efficacy in $\mathrm{CD}$ with an intensified regimen, as the disease was in remission (HBI 1-6 points) in most patients. Its safety profile was optimum as only one patient experienced an adverse reaction and withdrawal of the drug was not necessary.

\section{REFERENCES AND/OR ACKNOWLEDGEMENTS}

No conflict of interest.

\section{PSQ-061 ALEMTUZUMAB FOR RELAPSING-REMITTING MULTIPLE SCLEROSIS: EFFECTIVENESS AND SAFETY}

S Vázquez Blanco, S Fortes González* ${ }^{*}$ L Herrero Poch, JC De Miguel Bouzas, JM Castro Domínguez, L Villamayor Blanco.

\subsection{6/ejhpharm-2020-eahpconf.378}

Hospital Povisa, Pharmacy, Vigo, Spain

Background and importance Alemtuzumab is a humanised monoclonal antibody that selectively targets CD52, indicated in adult patients with relapsing-remitting multiple sclerosis (RRMS).

Aim and objectives To assess the effectiveness and safety of alemtuzumab for RRMS in the clinical setting.

Material and methods A retrospective observational study of all patients with RRMS treated with at least one course of alemtuzumab from July 2016 to March 2019 was carried out. The drug was administered by intravenous infusion on 5 consecutive days at baseline and on 3 consecutive days 12 months later. All patients received prophylaxis with methylprednisolone, antihistamines, antipyretics and acyclovir.

Alemtuzumab was started in adults with active disease, defined by clinical or imaging features despite the use of immunomodulating therapies, or having a fast and aggressive course. The variables studied were sex, age, time from diagnosis, expanded disability status scale (EDSS), previous treatment, number of cycles, adverse events (AE) and number of relapses 